Check for updates

Cite this: Chem. Commun., 2020, 56,6822

Received 20th April 2020, Accepted 14th May 2020

DOI: $10.1039 / \mathrm{d} 0 \mathrm{cc} 02842 \mathrm{~h}$

rsc.li/chemcomm

\section{Raman reporters derived from aryl diazonium salts for SERS encoded-nanoparticles $\dagger$}

\author{
Yun Luo, ${ }^{* a}$ Yu Xiao, ${ }^{a}$ Delphine Onidas, ${ }^{a}$ Laura lannazzo, ${ }^{a}$ Mélanie Ethève-Quelquejeu, (D) ${ }^{a}$ \\ Aazdine Lamouri, ${ }^{\mathrm{b}}$ Nordin Félidj, (D) ${ }^{\mathrm{b}}$ Samia Mahouche-Chergui, ${ }^{\mathrm{c}}$ Thibault Brulé, ${ }^{d}$ \\ Nathalie Gagey-Eilstein, ${ }^{e}$ Florence Gazeau (D) ${ }^{f}$ and Claire Mangeney (D) *a
}

\begin{abstract}
Surface-enhanced Raman scattering (SERS) tags are usually prepared by immobilizing Raman reporters on plasmonic nanoparticles (NPs) via thiol-based self-assembled monolayers. We describe here the first example of SERS tags obtained by combining gold NPs and aryl diazonium salts. This strategy results in robust $\mathrm{Au}-\mathrm{C}$ covalent bonds between the Raman reporter and the NPs, thus ensuring a high stability of the nanohybrid interface.
\end{abstract}

The popularity of surface-enhanced Raman scattering (SERS) tags has increased in recent decades, as an alternative to fluorescent probes, due to their attractive properties. ${ }^{1}$ Indeed, unlike the broad spectral feature of fluorescence probes, SERS tags provide multiple sets of narrow peaks, resulting in low spectral overlap and high multiplexing ability. Besides, a single light source can be used to excite multiple Raman reporters, with negligible photobleaching, low background and a high sensitivity that competes with that of fluorescence. Moreover, their low toxicity, compared to quantum dots, has boosted their attractiveness for in vitro and in vivo biosensing. ${ }^{2}$ SERS tags are based on several key elements: ${ }^{3}$ a metal core providing the source of SERS enhancement, ${ }^{4,5}$ an adsorbed Raman reporter molecule giving the spectral fingerprints, a protective shell to enhance the stability of the adsorbed reporter molecule and a targeting entity to improve specificity. The stacking of all these elements into a single SERS tag is a multi-step process which is usually challenging. ${ }^{6}$ In particular, reduction of the colloidal stability of plasmonic nanoparticles during the adsorption of Raman codes may lead to uncontrolled particle agglomeration.

\footnotetext{
${ }^{a}$ Université de Paris, LCBPT, UMR 8601, F-75006 Paris, France. E-mail: claire.mangeney@parisdescartes.fr, yun.sun@parisdescartes.fr

${ }^{b}$ Université de Paris, ITODYS, UMR 7086, 75013 Paris, France

${ }^{c}$ Université Paris-Est, ICMPE, UMR 7182, 94320 Thiais, France

${ }^{d}$ HORIBA France SAS, 14 Boulevard Thomas Gobert, Passage Jobin Yvon, 91120 Palaiseau, France

${ }^{e}$ Université de Paris, COMETE, CNRS UMR 8638, F-75006 Paris, France

${ }^{f}$ Université de Paris, MSC, CNRS UMR 7057, 75013 Paris, France

$\dagger$ Electronic supplementary information (ESI) available. See DOI: 10.1039/ $\mathrm{d} 0 \mathrm{cc} 02842 \mathrm{~h}$
}

To solve this problem, the use of polymers or the co-adsorption of small Raman reporters and long-chain thiols was proposed, preserving colloidal stability all along the codification process. ${ }^{7,8}$ However, the chemical stability of thiolate self-assembled monolayers (SAMs) is a critical issue for applications in ambient and aqueous environments. Indeed, several studies have shown that SAMs desorb spontaneously from the surface in aqueous solutions after only few days ${ }^{9-11}$ and degrade upon laser excitation. ${ }^{12}$ Significant progress in stability have been obtained by using longer-chain thiols, ${ }^{13}$ small amount of amphiphilic surfactant molecules ${ }^{10}$ or multidentate sulfur-based adsorbates. ${ }^{14}$ Nevertheless, the development of alternative surface chemical approaches enabling robust covalent attachment of Raman reporters to plasmonic NPs can be expected to result in more significant improvements of interfacial stability. Aryl diazonium salts have emerged in recent years as a new generation of surface modifiers for plasmonic nanoparticles, ${ }^{15-19}$ in alternative to thiol-based SAMs. These molecules present several advantages such as their ease of preparation, rapid grafting, large choice of reactive functional groups and robust metal-C interfacial bonds with the surface. Their grafting on the surface of plasmonic NPs is a two-stage process: ${ }^{20,21}$ (i) first, the spontaneous reduction of the diazonium salts generates an aryl radical that binds to the surface, and (ii) in a second step, due to their high reactivity, these radicals react with the first grafted layer to give nanometer to micrometer thick polyaryl layers. Aryl diazonium salts were used to modify plasmonic NPs in order to design NPs-coated electrodes ${ }^{22}$ colorimetric nanosensors ${ }^{23}$ antimicrobial agents ${ }^{24}$ and functional lithographic arrays. ${ }^{25-27}$ In the latter case, they were shown to result in regioselective grafting of organic patches located specifically in the regions of maximum near field enhancement around nanoparticles, via site-selective hotelectron mediated reduction of aryl diazonium salts. However, the application of these surface agents as Raman reporters for SERS encoded-nanoparticles has never been reported so far, although aryl layers derived from diazonium salts were shown to exhibit intense Raman signals. ${ }^{28,29}$ In this work, we explore the potential of aryl diazonium salts to provide a new class of 

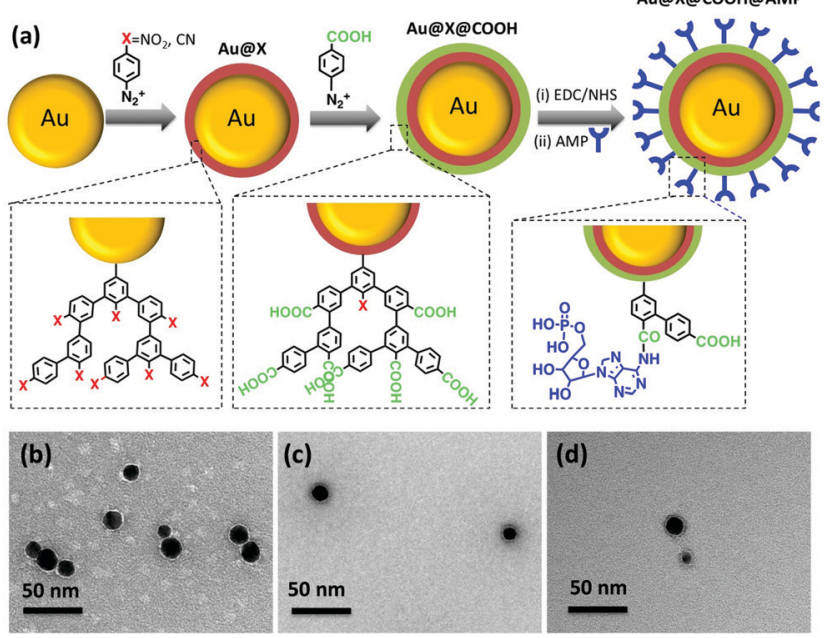

Fig. 1 (a) Schematic representation of the key steps for the preparation of SERS-encoded NPs using aryl diazonium salts. The Raman reporter groups $\left(-\mathrm{NO}_{2},-\mathrm{CN}\right)$ are grafted onto gold NPs via $\mathrm{Au}-\mathrm{C}$ covalent bonds. A layer bearing $-\mathrm{COOH}$ groups is then added for post-functionalization with a nucleotide, here AMP. Typical TEM images of (b) AuNPs, (c) Au@CN@$\mathrm{COOH}$ aAMP (d) Au@NO $\mathrm{aCOOH}_{2} \mathrm{AMP}$

Raman reporters with several advantages, such as: (i) the formation of strong interfacial bonds with the supporting NPs, (ii) the presence of intense SERS fingerprints, (iii) the possibility to create multilayers with various functions. The formation of multilayers will be exploited here to introduce straightforwardly (i) several SERS labels along the grafted polyaryl chains and (ii) postfunctionalization moieties at their end. As a proof of concept, we selected three different aryl diazonium salts. Two of them, bearing either nitro groups (4-nitrobenzenediazonium tetrafluoroborate, noted $\mathrm{D}-\mathrm{NO}_{2}$ ) or cyano groups (4-cyanobenzenediazonium tetrafluoroborate, noted D-CN) were chosen to act as Raman reporters. The third one possesses carboxyl-terminated groups (4-carboxybenzene diazonium tetrafluoroborate, noted $\mathrm{D}-\mathrm{COOH}$ ) for post-functionalization with the nucleotide adenosine 5 '-monophosphate (AMP) (see Fig. 1). Adenosine derivatives are important intermediary metabolites in cells, which have fundamental impacts on a wide range of cellular processes. ${ }^{30}$ Particularly, AMP was identified recently to be a novel tumour-targeting ligand, guiding nanoparticles to cancer cells via adenosine A1 receptor, offering promising prospects for clinical diagnostic and therapeutic applications. $^{31}$ The functionalization of spherical gold NPs by the three aryl diazonium salts $\left(-\mathrm{NO}_{2},-\mathrm{CN},-\mathrm{COOH}\right)$ and AMP was performed in water at room temperature, providing a simple method to create multifunctional SERS tags. The modified NPs were characterized by transmission electron microscopy (TEM), UV-vis absorption, X-ray photoelectron spectroscopy (XPS) and SERS.

Spherical AuNPs with an average diameter of $15 \pm 3 \mathrm{~nm}$ (see typical TEM image Fig. 1b), were prepared through the wellknown Turkevich method, ${ }^{32}$ by citrate reduction of $\mathrm{HAuCl}_{4}$. The procedure for grafting the Raman reporters on the AuNPs consisted to add the corresponding diazonium salts $\left(5 \times 10^{-5} \mathrm{M}\right)$ to the AuNPs aqueous dispersions ([AuNPs] $=8 \times 10^{-9} \mathrm{M}$ ) and to let them react spontaneously during $60 \mathrm{~min}$ at room temperature.
Multilayers were obtained by adding the diazonium salts successively, one after the other in a controlled sequence. The functionalized AuNPs were then purified through 3 cycles of centrifugation/ redispersion in water. Post-functionalization with AMP was performed by mixing an aqueous AMP solution $\left(10^{-4} \mathrm{M}\right)$ with the resulting NPs, and adding 1-ethyl-3-(3-dimethylaminopropyl)carbodiimide (EDC) and $N$-hydroxysuccinimide (NHS), in the presence of $0.001 \mathrm{wt} \%$ polyvinylpyrrolidone (PVP), as a stabilizer. After reaction at $4{ }^{\circ} \mathrm{C}$ overnight, the particles were washed through 3 cycles of centrifugation/redispersion in water. Details on the functionalization experiments are given in ESI. $\dagger$ Transmission electron microscopy (TEM) images (see Fig. 1b-d and Fig. S1, ESI $\dagger$ ) show individually dispersed particles indicating that the reaction of the AuNPs with the diazonium salts does not disturb their colloidal stability. Interestingly, it can be seen from TEM images that the functionalization process leads to the appearance of a thin layer covering the NPs, likely due to the presence of the polyaryl layers and AMP. An average thickness of the organic coating of $c a$. 3-4 nm was estimated using 100 NPs from different TEM images (see Table S1, ESI $\dagger$ ). The observation of polyaryl layers around the gold cores can be explained by the grafting reaction mechanism. Indeed, it is based on the formation of aryl radicals by spontaneous cleavage of diazohydroxides $\mathrm{Ar}-\mathrm{N}=\mathrm{N}-\mathrm{OH}$ that form from aryl diazonium salts in neutral aqueous media, as described previously. ${ }^{33}$ The formed aryl radicals bind to the AuNPs surface and to the first (and further) grafted aromatic groups via homolytic aromatic substitution, leading to polyaryl layers. The extinction profile of the AuNPs (Fig. 2a and b) retained its original shape upon functionalization confirming that the colloidal stability was preserved. Nevertheless, a progressive red shift of the plasmon band was observed after each functionalization step (see zoom in Fig. 2a and $\mathrm{b}$ and $\lambda_{\max }$ values in Table S2, ESI $\dagger$ ). This red shift increases with the number of grafted layers and reaches ca. $10 \mathrm{~nm}$ for AMP-modified NPs, likely due to the modification of the local dielectric environment around the $\mathrm{Au}$ cores.

It is noteworthy that the addition of a small amount of PVP $(0.001 \mathrm{wt} \%)$ in the colloidal dispersion at the post-functionalization step was necessary in order to maintain a perfect colloidal stability, as described in ESI $\dagger$ (see Fig. S2a). However, this parameter should be carefully controlled to ensure that the NP surface is still accessible for post-functionalization with AMP. Indeed, above a
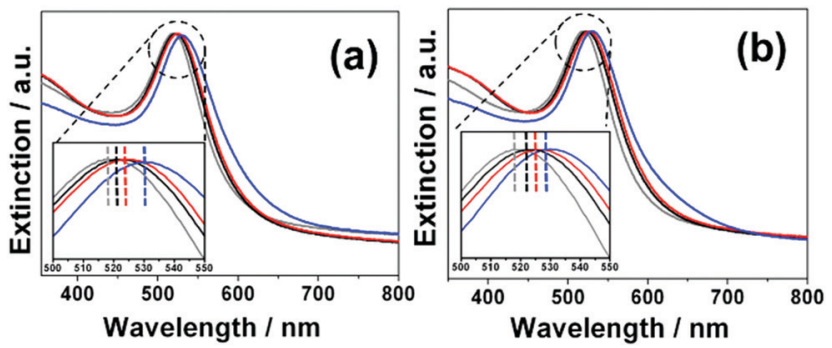

Fig. 2 (a) Normalized extinction spectra of AuNPs (grey), Au@CN (black), AuaCNaCOOH (red) and AuaCNaCOOH@AMP (blue); (b) normalized extinction spectra of AuNPs (grey), Au@NO (black), Au@NO $\mathrm{NO}_{2} \mathrm{aCOOH}$ (red) and Au@NO 2 aCOOH@AMP (blue). 
certain [PVP] value, the grafting of AMP is no more detected by SERS after the post-functionalization step (see Fig. S2b, ESI $\dagger$ ). The stability of the nanoprobes was evaluated in various media: aqueous solutions with a high ionic force, acid or basic solutions. No sign of aggregation could be detected, as shown in Fig. S3 (ESI $\dagger$ ), regardless the medium to which the particles were exposed, demonstrating an extended colloidal stability. The surface chemical composition of the modified AuNPs was investigated by XPS (see Table S3 and Fig. S4, ESI $\dagger$ ). The XPS spectra revealed large modifications after functionalization and confirmed the successful covering of the gold cores by the functional polyaryl layers and AMP.

The SERS properties of the encoded particles were studied at $633 \mathrm{~nm}$ laser excitation and compared to the Raman spectra of the free diazonium salts (Fig. S5, ESI $\dagger$ ). Several key features can be pointed out: first, the intense $\mathrm{N} \equiv \mathrm{N}$ stretching peak, observed in the Raman spectra of the diazonium salt powders at ca. 2280-2300 $\mathrm{cm}^{-1}$ disappears completely for all nanoprobes, confirming the removal of the diazonium group during the grafting reaction. At the same time, one notices the appearance of a small peak at $c a .410-416 \mathrm{~cm}^{-1}$, assigned to the $\mathrm{Au}-\mathrm{C}$ stretching vibration, evidencing the covalent grafting of the aryl layers. The SERS spectra display also the characteristic signature of phenyl-derivatives stemming from diazonium salts, particularly the aryl ring stretching peak at $c a$. 1580-1595 $\mathrm{cm}^{-1}$. For the nanoprobes $\mathrm{Au} @ \mathrm{NO}_{2}$ and $\mathrm{Au} @ \mathrm{CN}$, the characteristic $\mathrm{NO}_{2}$ and CN stretching modes appear at $1330 \mathrm{~cm}^{-1}$ and $2220 \mathrm{~cm}^{-1}$, respectively. Interestingly, thanks to the radical coupling mechanism in diazonium salt chemistry, different functional layers could be grafted one after another around the gold core, forming multifunctional layers with a wide variety of SERS signatures. For example, Fig. 3 compares the SERS spectra and the corresponding Raman images of Au@CN, Au@NO,$A u @ N_{2} @ C N$ and $\mathrm{Au@}$ $\mathrm{CN} @ \mathrm{NO}_{2}$, deposited as dried drops on a glass slide. Each type of NPs reveals distinct characteristic spectra, which can be associated to one particular color in Raman imaging. The spectra of Au@CN are associated here to the magenta color, $\mathrm{Au} @ \mathrm{NO}_{2}$ to the blue, $\mathrm{Au} @ \mathrm{NO}_{2} @ \mathrm{CN}$ to the green and Au@CN@NO $\mathrm{N}_{2}$ to the red. The blackline corresponds to the glass slide.

A layer of carboxyl-terminated functions was then added to the nanoconstructs to obtain a post-functionalizable platform. The successive grafting of the different layers for Au@CN@COOH and $\mathrm{Au} @ \mathrm{NO}_{2} @ \mathrm{COOH}$ could be assessed by following the increase in

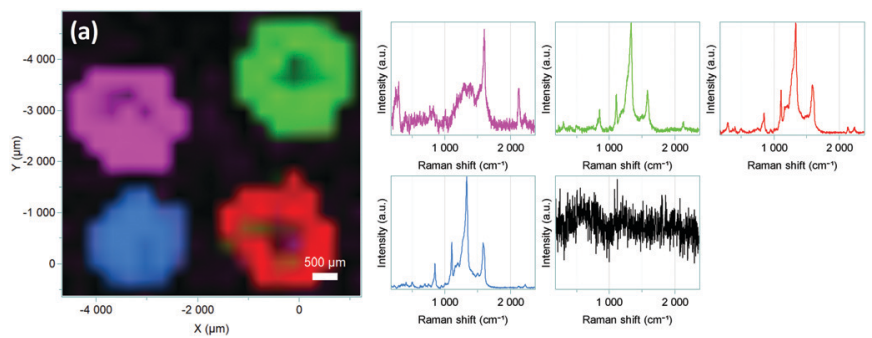

Fig. 3 Raman images and corresponding SERS spectra of Au@CN (magenta),

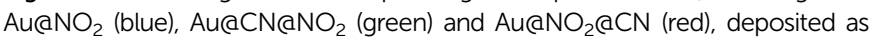
dried drops on a glass slide.

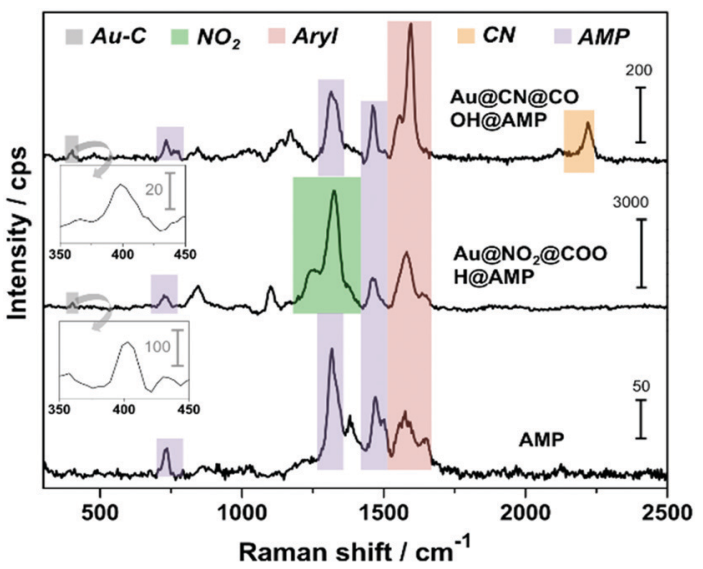

Fig. 4 SERS spectra of AuaCNaCOOH@AMP and AuaNO 2 aCOOHaAMP. The Raman spectrum of AMP is used as a reference. Inset: Zoom in the spectral range $350-450 \mathrm{~cm}^{-1}$, revealing the presence of $\nu_{\mathrm{Au}-\mathrm{C}}$ peak.

intensity of the aryl ring stretching peak, $I_{\text {aryl }}$ at $1580-1595 \mathrm{~cm}^{-1}$ (see Fig. S6 and S7, ESI $\dagger$ ) and the evolution of $I_{\mathrm{CN}} / I_{\mathrm{Au}-\mathrm{C}}, I_{\mathrm{NO}_{2}} / I_{\mathrm{Au}-\mathrm{C}}$ and $I_{\text {aryl }} / I_{\mathrm{Au}-\mathrm{C}}$ band intensity ratios (see Fig. S8, ESI $\dagger$ ). An activation step using EDC/NHS was necessary to activate the carboxyl groups for direct conjugation to the nucleotide AMP, via amide bonds. The presence of the grafted AMP generates new SERS characteristic peaks $^{34}$ (see Fig. 4) at $\sim 733 \mathrm{~cm}^{-1}$ (in-plane ring breathing of adenine ring), $\sim 1332 \mathrm{~cm}^{-1}$ (ring stretching mode) and $\sim 1460 \mathrm{~cm}^{-1}$ (C-N stretching).

The other peaks remain unchanged demonstrating the robustness of the grafted layers derived from diazonium salts at the surface of the nanoconstruct. This new generation of SERS encoded NPs, based on AuNPs functionalized by aryl diazonium salts, was then evaluated as labels for Raman bioimaging inside cells (see Fig. 5).

Proof of concept experiments consisted to expose different cell lines (EGI-1 and CT-26 cells) to Au@CN@COOH@AMP and $\mathrm{Au} @ \mathrm{NO}_{2} @ \mathrm{COOH} @ A M P$ (details of the experiments are given in ESI $\dagger$ ). Fig. 5 shows that, when internalized inside cells, the SERS tags could be perfectly detected as bright spots, probably due to accumulation into endosomal compartments. These results demonstrate that this new generation of SERS labels offers promising prospects for multiplex SERS-based Raman imaging.

In summary, Raman reporters derived from aryl diazonium salts were grafted on gold NPs to design a new generation of SERS encoded-nanoparticles. AuNPs were modified by three different aryl diazonium salts, bearing either nitro or cyano groups and carboxyl-terminated function. These nanoprobes were shown to combine unique SERS spectra and post-functionalization capacity for the immobilization of the nucleotide AMP. Unlike thiol-derived Raman reporters forming monolayers around plasmonic NPs, this approach enables the formation of strongly attached multilayers around the gold cores, with various chemical functions, providing a breakthrough strategy to create multifunctional SERS tags. It thus offers several advantages over conventional methods of SERS tag preparation, such as: (i) the formation of strong covalent bonds between the AuNP 


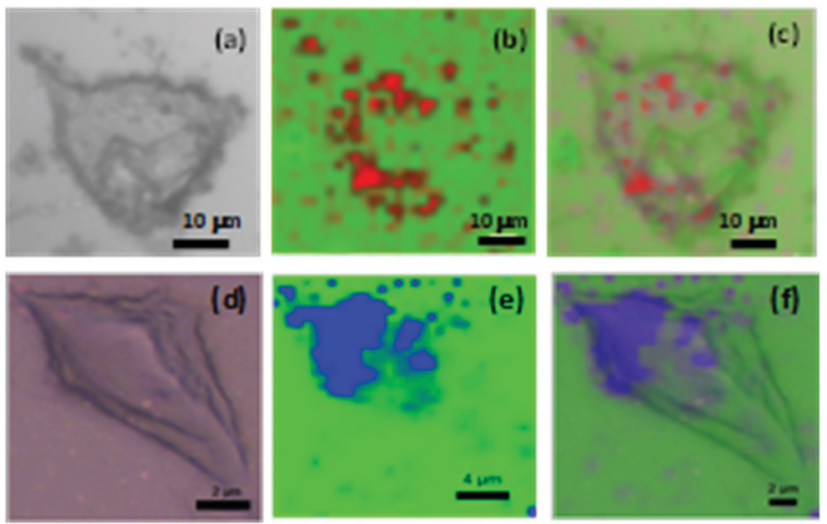

Fig. 5 ( $a$ and d) Bright-field optical images and ( $b$ and e) Raman images of EGl-1 cells after uptake of Au@CNaCOOH@AMP, observed as red spots (b) and CT26 after uptake of $\mathrm{AuaNO} \mathrm{N}_{2} \mathrm{ACOOH}$ aAMP, observed as blue spots (e). (c) Overlay images of (a) and (b); (f) overlay images of (d) and (e), revealing the intracellular distribution of the SERS tags inside cells. The excitation laser wavelength was $633 \mathrm{~nm}\left(1 \mathrm{~mW}\right.$, grating $300 \mathrm{~g} \mathrm{~mm}^{-1}$ objective $100 \times$ hole $50 \mu \mathrm{m}$, acq. time $2 \mathrm{~s}$ per pt, $X Y$ parameters: $10^{4} \times 10^{9}$ $0.3 \times 0.3 \mu \mathrm{m}$ per pt).

surface and the Raman labels and (ii) the growing of multifunctional layers around the gold cores, using a simple and straightforward process (in air, at room temperature). Therefore, this aryl diazonium salt-based approach will not only pave a new way for the functionalization of AuNPs by multilayers but also provide a general strategy to design SERS-encoded NPs.

The authors thank G. Pehau-Arnaudet (Institut Pasteur) for TEM analysis, Horiba for Raman imaging, L. Fouassier (Sorbonne Université) and A. Nicolas Boluda (Univ. de Paris) for providing cells.

\section{Conflicts of interest}

There are no conflicts to declare.

\section{Notes and references}

1 L. Fabris, ChemNanoMat, 2016, 2, 249.

2 Y. Wang, B. Yan and L. Chen, Chem. Rev., 2013, 113, 1391.

3 R. A. Alvarez-Puebla and L. M. Liz-Marzán, Small, 2010, 6, 604.

4 M. Tabatabaei, D. McRae and F. Lagugné-Labarthet, Recent advances of plasmon-enhanced spectroscopy at bio-Interfaces. Frontiers of Plasmon Enhanced Spectroscopy, 2016, vol. 1246, pp. 183-207.

5 Y. B. Rus, L. Galmiche, P. Audebert, A. Courty, E. Maisonhaute and F. Miomandre, ChemistrySelect, 2019, 4, 1298.

6 L. A. Lane, X. Qian and S. Nie, Chem. Rev., 2015, 115, 10489.
7 E. Lenzi, D. Jimenez de Aberasturi and L. M. Liz-Marzán, ACS Sens., 2019, 4, 1126; J. Gao, X. Huang, H. Liu, F. Zan and J. Ren, Langmuir, 2012, 28, 4464.

8 B. Mir-Simon, I. Reche-Perez, L. Guerrini, N. Pazos-Perez and R. A. Alvarez-Puebla, Chem. Mater., 2015, 27, 950.

9 N. Bhatt, P.-J. J. Huang, N. Dave and J. Liu, Langmuir, 2011, $27(10), 6132$.

10 G. Yang, N. A. Amro, Z. B. Starkewolfe and G.-Y. Liu, Langmuir, 2004, 20, 3995.

11 C. Vericat, M. E. Vela, G. Benitez, P. Carro and R. C. Salvarezza, Chem. Soc. Rev., 2010, 39, 1805.

12 J. Walia, J.-M. Guay, O. Krupin, F. Variola, P. Berini and A. Weck, Phys. Chem. Chem. Phys., 2018, 20, 238.

13 S.-W. Tam-Chan, H. A. Biebuyck, G. M. Whitesides, N. Jeon and R. G. Nuzzo, Langmuir, 1995, 11, 4371.

14 P. Chinwangso, A. C. Jamison and T. R. Lee, Acc. Chem. Res., 2011, 44, 511.

15 L. Laurentius, S. R. Stoyanov, S. Gusarov, A. Kovalenko, R. B. Du, G. P. Lopinski and M. T. McDermott, ACS Nano, 2011, 5, 4219.

16 F. Mirkhalaf, J. Paprotny and D. J. Schiffrin, J. Am. Chem. Soc., 2006, 128, 7400 .

17 R. Ahmad, N. Felidj, L. Boubekeur-Lecaque, S. Lau-Truong, S. GamDerouich, P. Decorse, A. Lamouri and C. Mangeney, Chem. Commun., 2015, 51, 9678.

18 L. Troian-Gautier, H. Valkenier, A. Mattiuzzi, I. Jabin, N. Van den Brande, B. Van Mele, J. Hubert, F. Reniers, G. Bruylants, C. Lagrost and Y. Leroux, Chem. Commun., 2016, 52, 10493.

19 M. Bouriga, M. M. Chehimi, C. Combellas, P. Decorse, F. Kanoufi, A. Deronzier and J. Pinson, Chem. Mater., 2013, 25, 90.

20 J. Pinson and F. Podvorica, Chem. Soc. Rev., 2005, 34, 429.

21 A. Berisha, M. M. Chehimi, J. Pinson and F. I. Podvorica, Electrode surface modification using diazonium salts, ed. A. J. Bard and C. G. Zoski, Electroanalytical Chemistry, CRC Press, Boca Raton, FL, 2016, vol. 26.

22 E. Gervais, Y. Aceta, P. Gros and D. Evrard, Electrochim. Acta, 2018, $261,346$.

23 J. Du, H. Du, X. Li, J. Fan and X. Peng, Sens. Actuators, B, 2017, 248, 318.

24 Y. Kalachyova, A. Olshtrem, O. A. Guselnikova, P. S. Postnikov, R. Elashnikov, P. Ulbrich, S. Rimpelova, V. Švorčík and O. Lyutakov, ChemistryOpen, 2017, 6, 254-260.

25 M. Nguyen, I. Kherbouche, S. Gam-Derouich, I. Ragheb, S. LauTruong, A. Lamouri, G. Levi, J. Aubard, P. Decorse, N. Felidj and C. Mangeney, Chem. Commun., 2017, 53, 11364.

26 M. Nguyen, A. Lamouri, C. Salameh, G. Levi, J. Grand, L. BoubekeurLecaque, C. Mangeney and N. Felidj, Nanoscale, 2016, 8, 8633.

27 V.-Q. Nguyen, Y. Ai, P. Martin and J.-C. Lacroix, ACS Omega, 2017, 2, 1947.

28 I. Tijunelyte, I. Kherbouche, S. Gam-Derouich, M. Nguyen, N. LidgiGuigui, M. Lamy de la Chapelle, A. Lamouri, G. Lévi, J. Aubard, A. Chevillot-Biraud, C. Mangeney and N. Felidj, Nanoscale Horiz., 2018, 3, 53.

29 M. Supur, S. R. Smith and R. L. McCreery, Anal. Chem., 2017, 89, 6463.

30 L. Antonioli, C. Blandizzi, P. Pacher and G. Hasko, Nat. Rev. Cancer, 2013, 13, 842.

31 T. Dai, N. Li, F. Han, H. Zhang, Y. Zhang and Q. Liu, Biomaterials, 2016, 83, 37.

32 J. Turkevich, P. C. Stevenson and J. A. Hillier, Discuss. Faraday Soc., 1951, 11, 55.

33 A. Berisha, C. Combellas, F. Kanoufi, P. Decorse, N. Oturan, J. Médard, M. Seydou, F. Maurel and J. Pinson, Langmuir, 2017, 33, 8730.

34 J. Kundu, O. Neumann, B. G. Janesko, D. Zhang, S. Lal, A. Barhoumi, G. E. Scuseria and N. J. Halas, J. Phys. Chem. C, 2009, 113, 14390. 